\title{
An outbreak of acute haemorrhagic conjunctivitis in Kaduna, Nigeria
}

\author{
O E Babalola, S S Amoni, E Samaila, U Thaker, S Darougar
}

\begin{abstract}
Clinical studies were carried out on two groups of patients with acute haemorrhagic conjunctivitis (AHC) during an epidemic in 1985 in Northern Nigeria. Group 1 consisted of 99 students attending a girls' boarding school, group 2 of 200 patients selected randomly from 1000 examined at the local clinic.

Moderate to severe hyperaemia and papillary responses were present in the palpebral conjunctiva of all patients, and $234(66 \%)$ had subconjunctival haemorrhages. Transient superficial punctate keratitis was noted in over $60 \%$ of patients. A transient flare suggestive of a low grade iritis was seen in five patients. No neurological disorders were noted.

Serological studies were carried out on patients from group 2. Fifteen paired and 20 single serum samples were titrated against adenovirus type 4 (Ad-4) and enterovirus type 70 (EV-70). Two pairs of sera showed a 4-fold rise in antibody levels to $\mathrm{EV}-70$, whereas the antibody titres to EV-70 in the rest of the sera ranged from 1:20 (no antibody) to $1: 160$. None of the paired serum samples showed a 4-fold rise in antibody levels to adenovirus.

The results of clinical studies and serological findings support EV-70 as a probable cause of AHC in Nigeria.
\end{abstract}

Acute haemorrhagic conjunctivitis (AHC) was first reported in Ghana, in 1969,12 and was dubbed 'Apollo' disease by the local populace because it coincided with Apollo II landing on the moon. AHC epidemics were later reported in most developed and developing countries. ${ }^{3}$ In Nigeria, AHC occurred in epidemic proportions at about the same time as it was first reported in Ghana ${ }^{45}$ Enterovirus 70, a picornavirus, has been isolated from most cases of AHC, but Coxsackie A24 and adenovirus have also been isolated during AHC epidemics. ${ }^{67}$ In Nigeria attempts to isolate the cause of AHC during an epidemic in 1981 were unsuccessful, but serological tests indicated that the epidemic was probably due to enterovirus $70 .{ }^{8}$

We report here the results of clinical and serological studies carried out during an epidemic of AHC in Northern Nigeria in 1985. In this study no attempt was made to perform isolation tests because of the lack of facilities.

\section{Patients and methods}

The first cases of AHC were referred from a girl's boarding school in Kaduna metropolis. A team was sent to the school to investigate, and a total of 116 students were examined. Inquiries were made as to the age, time of onset, symptoms, and conditions in the living quarters. Laterality, visual acuity, conjunctival signs, corneal involvement, preauricular lymphadenopathy, and other associated signs were assessed. At about this time an epidemic of AHC developed in the town and the adjacent districts. Of patients attending the Guinness Ophthalmic Unit of the Ahmadu Bello University with AHC one in five were selected for entry into this study. Basic epidemiological data, such as history of illness, exposure to infection and previous treatment were recorded in a special proforma. Ocular examination was carried out with a slit-lamp, and attention was paid to the location of subconjunctival haemorrhages, superficial punctate keratitis (SPK), keratic precipitates, and coalescent corneal lesions. The anterior chamber and pupils were examined, and neurological disorders were looked for. Patients were asked to return on the third day, and first, second, and third weeks after the first presentation.

\section{SEROLOGY}

Blood was collected by venepuncture from 35 patients at first presentation and from 15 seven days afterwards. Serum was separated from these samples on the same day and kept frozen until sent to the laboratory in Britain.

\section{ANTIGENS}

The Singapore strain (EH 91/71) and the London strain $(23356 / 71)$ were used as antigens for the detection of antibodies against enterovirus type 70 (EV-70). Adenovirus type 4 (Ad-4) was used as antigen for the detection of group specific antiadenovirus antibody. Secondary baboon kidney cells (BK) were used for propagation of EV-70 strains. The single strength Wellcome 199 medium was supplemented with $10 \%$ inactivated fetal bovine serum (FBS), $0.1 \mathrm{~g} / 1$ glucose, $25 \mathrm{ml} / 1$ of $4.4 \%$ sodium bicarbonate, and antibiotics (penicillin at 100 units $/ \mathrm{ml}$ and streptomycin at $100 \mu \mathrm{g} / \mathrm{ml})$. HEp-2 cells were used for propagation of Ad-4. The cells were grown in growth medium (GM), which consisted of Eagles' minimum essential medium (MEM) supplemented with glutamine, vitamins, $10 \%$ FBS, and antibiotics (vancomycin $100 \mu \mathrm{gm} / \mathrm{ml}$ and streptomycin $50 \mu \mathrm{gm} / \mathrm{ml}$ ). Confluent monolayers were maintained on maintenance medium (MM), which was similar to GM but with only 3\% FBS.

Monolayers of BK and HEp-2 cells in $75 \mathrm{~cm}^{2}$ flasks were drained of growth medium and inoculated with $5 \mathrm{ml}$ of appropriate seed of virus culture (propagated and titrated previously). The virus was adsorbed on to the monolayer for one hour at $35^{\circ} \mathrm{C}$. The inoculum was removed
Correspondence to: Professor $S$ Darougar. 
and replaced with prewarmed fresh $M M$ and the flasks were further incubated at $35^{\circ} \mathrm{C}$. Between 50 and $75 \%$ of the cytopathic effect was usually observed within 24 to 48 hours after infection. The monolayers were then washed twice with phosphate buffered saline at pH $7 \cdot 3$ (PBS). Next, the cells were scraped into PBS, centrifuged at $300 \mathrm{~g}$ for 10 minutes at room temperature, and the pellet was finally suspended in fresh PBS to give a turbid preparation. Controls were prepared in a similar way but from uninfected cells.

The cell suspension was then dotted on glass slides with mapping pens (Joseph Gillott Co. pen no. 659). Five antigen dots consisting of (i) uninfected BK cells (as negative control); (2) BK cells infected with $23356 / 71$; (3) BK cells infected with EH91/71; (4) uninfected HEp-2 cells (negative control); and (5) HEp-2 cells infected with Ad-4, were prepared in a cluster. Each slide contained eight clusters. The slides were dried and fixed in acetone for 10 minutes at room temperature and stored at $-20^{\circ} \mathrm{C}$ until used.

Dilutions of test serum (1:20 to $1: 640)$ were each applied on one cluster, and the slides were incubated in a humid chamber at $35^{\circ} \mathrm{C}$ for 30 minutes. The slides were then washed in PBS at pH 7.3 for 15-20 minutes, and fluorescein isothiocyanate (FITC) labelled goat antihuman IgG serum was applied as a second antibody layer and incubated as above. They were then washed in PBS for 10 minutes and for a further five minutes in deionised water. After being dried they were mounted in buffered glycerol. Each dot of antigen was examined with a Zeiss standard $18 \mathrm{UV}$ microscope fitted with a filter set 10 under 400 times magnification for the presence of brightly fluorescing particulated inclusions in the cytoplasm of infected cells. The titre of the serum was defined as the reciprocal of the test dilution which gave $25-50 \%$ fluorescence. A negative and a positive control serum were always included to ensure specificity of the slide antigen.

\section{Results}

BOARDING SCHOOL

AHC was detected in 99 students ranging in age from 8 to 19 years. The cases were detected from at least seven different dormitories, most being from one dormitory. All the dormitories were overcrowded and short of water. It was found that the students were commonly using each others' towels, handkerchiefs, and cosmetics. AHC was bilateral in 78 cases $(79 \%)$. The visual acuity was $6 / 6$ in 125 eyes. The symptoms and signs of AHC in these students are presented in Tables 1 and 2.

Ophthalmic clinic. About 1000 cases of AHC presented at the clinic between July and October 1985. Two hundred consecutive cases (one in every five examined) were selected and examined in detail.

Age and sex. The ages of patients varied from 9 to 75 years, mean 26.4 years. Altogether there were 89 females $(44 \cdot 5 \%)$ and 111 males $(55 \cdot 5 \%)$.

Ethnicicity. $38.5 \%$ of the cases were Hausas, which forms the main ethnic group in the clinic's catchment area. Yorubas accounted for $16 \%$ and
TABLE I Symptoms at first examination in 177 infected eyes of 99 students

\begin{tabular}{ll}
\hline Symptom & Number $(\%)$ \\
\hline Pain & $127(72)$ \\
Itching & $31(17)$ \\
Lacrimation & $23(13)$ \\
Foreign body sensation & $23(13)$ \\
Redness & $22(12)$ \\
Discharge & $5(3)$ \\
Reduced visual acuity & $4(2)$ \\
Swelling & $2(1)$ \\
Headache & $1(0 \cdot 5)$ \\
\hline
\end{tabular}

TABLE II Signs at first examination in 177 infected eyes of 99 students

\begin{tabular}{ll}
\hline Sign & Number $(\%)$ \\
\hline Hyperaemia & $177(100)$ \\
Papillary hypertrophy & $96(54)$ \\
Subconjunctival haemorrhage & $81(46)$ \\
Watery discharge & $154(87)$ \\
Mucopurulent discharge & $23(13)$ \\
Gross lid oedema & $7(4)$ \\
\hline
\end{tabular}

TABLE III Occupational groupings in 200 AHC clinical cases

\begin{tabular}{ll}
\hline Occupation & Number $(\%)$ \\
\hline Students & $51(25 \cdot 5)$ \\
Industrial workers & $49(24 \cdot 5)$ \\
Housewives & $41(20 \cdot 5)$ \\
Office workers & $34(17)$ \\
Military/paramilitary & $8(4)$ \\
Children & $8(4)$ \\
Others & $6(3)$ \\
Unstated & $3(1 \cdot 5)$ \\
\hline
\end{tabular}

TABLE IV Previous medication before consultation in 200 AHC clinical cases

\begin{tabular}{ll}
\hline Medication & Number $(\%)$ \\
\hline Nil & $108(54 \cdot 0)$ \\
Antibiotic drops & $35(17 \cdot 5)$ \\
Unspecified drops & $13(6 \cdot 5)$ \\
Does not know & $10(5 \cdot 0)$ \\
Steroid containing drops & $7(3 \cdot 5)$ \\
Sugar water & $6(3 \cdot 0)$ \\
Mentholatum & $2(1 \cdot 0)$ \\
Hot fomentation & $1(0 \cdot 5)$ \\
Ice blocks & $1(0 \cdot 5)$ \\
Irrigation & $1(0 \cdot 5)$ \\
Local gin & $1(0 \cdot 5)$ \\
Atropine & $1(0 \cdot 5)$ \\
Yeast tablets & $1(0 \cdot 5)$ \\
Unstated & $13(6 \cdot 5)$ \\
\hline
\end{tabular}

TABLE V Main symptoms in 200 AHC clinical cases

\begin{tabular}{ll}
\hline Complaint & Number $(\%)$ \\
\hline Discharge & $195(97 \cdot 5)$ \\
Foreign body sensation & $182(91 \cdot 0)$ \\
Pain & $170(85 \cdot 0)$ \\
Blurred vision & $84(42 \cdot 0)$ \\
\hline
\end{tabular}

Nupes for $5 \cdot 5 \%$. There were nine Ibos $(4 \cdot 5 \%)$ and nine Idomas $(4 \cdot 5 \%)$. The others belonged to minority groups.

Occupation. Distribution of AHC in relation to occupation is shown in Table 3. Although the Nigerian population is predominantly agrarian, only one farmer was seen with AHC. Five patients belonged to the executive/managerial group, suggesting that the disease was mainly confined to workers with low income.

Previous treatment. Ninety-two (46\%) of the two hundred patients had received topical medication prior to consultation. The drugs were obtained mainly from chemists, dispensaries, and shops without prescription. The types of medication used are shown in Table 4. One patient had applied a mixture of locally brewed gin, 'ogogoro', and sugar water, ending 
up with severe corneal melting and corneal vascularisation.

Previous conjunctivitis. In $136(68 \%)$ of the patients a previous history of severe conjunctivitis was reported.

Incubation period. The incubation period varied widely, but about $55 \%$ of cases had contracted the disease within one week of exposure.

Laterality. One hundred and forty patients (70\%) attended the clinic by the second day of the onset of conjunctivitis. The condition was bilateral in $154(77 \%)$ cases and unilateral in 46 (23\%) cases. In $132(86 \%)$ of the bilateral cases the second eye developed infection within 24 hours of the first eye. For the rest it varied from two to seven days.

Symptoms. The main symptoms are shown in Table 5.

Signs. The main clinical signs are shown in Table 6. Of 354 infected eyes $250(70.6 \%)$ had a visual acuity of $6 / 9$ or better. In 23 patients the vision was less than $6 / 60$. The causes of visual defects in these patients are shown in Table 7. Moderate to severe hyperaemia and papillary responses were present in the palpebral conjunctiva of all patients, and $234(66 \%)$ had subconjunctival haemorrhages.

In $189(53.4 \%)$ of the affected eyes the corneal involvement was usually in the form of superficial punctate keratitis, and in $20(5 \cdot 7 \%)$ coalescent corneal erosions were present. Anterior chamber flare was found in association with AHC in five cases. The flare resolved following improvement in the conjunctivitis. Complications due to secondary bacteria was not noted.

Treatment. Most of the patients were treated with sulphacetamide $10 \%$ eye drops four times a day and terramycin eye ointment at night. This was aimed at preventing secondary bacterial infection. Treatement was usually as an outpatient, but patients who had corneal lesions due to noxious topical self-medication were admitted to the hospital.

Follow-up. Only 69 patients came back after the first visit. Of the 40 patients seen on the third day only one was totally cured. Thirty-seven patients were seen after one week, of whom 12 (32\%) were cured. Eleven still had SPK, six had haemorrhages, and four complained of foreign body sensations. Thirteen patients were seen during the second week, of whom eight $(61 \%)$ were cured. One patient still had SPK, one complained of itching, and one of lacrimation. The others had minor symptoms. Only four patients came back for the third week follow-up. All were virtually cured except for one who had residual follicles.

\section{SEROLOGY}

Paired sera were collected from 15 patients. Twenty single serum samples were also collected during the visit.

The antibody levels to EV-70 and adenovirus in the 35 sera collected at the first visit ranged from $<1: 20$ (no antibody) to 1:160 (Table 8). It was interesting to note that sera which had a titre greater than 1:40 to EV-70 had a titre of $<1: 20$ to adenovirus and vice versa. There was no
TABLE VI Clinical signs in 354 affected eyes of 200 patients

\begin{tabular}{ll}
\hline Signs & Number $(\%)$ \\
\hline Bulbar Hyperaemia & $335(94 \cdot 6)$ \\
Discharge & $291(82 \cdot 2)$ \\
- Watery & $159(44 \cdot 9)$ \\
- Watery + mucopurulent & $180(50 \cdot 8)$ \\
- Mucopurulent & $84(23 \cdot 7)$ \\
Subconjunctival haemorrhage & $234(66 \cdot 1)$ \\
- Generalised & $127(35 \cdot 9)$ \\
- Superior fornix & $104(29 \cdot 4)$ \\
- Nasal & $2(0 \cdot 57)$ \\
- Temporal & $1(0 \cdot 28)$ \\
Superficial punctate keratitis & $189(53 \cdot 4)$ \\
Gross chemosis & $56(15 \cdot 8)$ \\
Preauricular lymphadenopathy & $24(6 \cdot 8)$ \\
Gross lid oedema & $19(5 \cdot 4)$ \\
Coalescent corneal erosions & $20(5 \cdot 7)$ \\
Anterior chamber flare & $5(1 \cdot 4)$ \\
\hline
\end{tabular}

TABLE VII Causes of visual defects in 23 patients with AHC

\begin{tabular}{ll}
\hline Findings & Frequency \\
\hline Pre existent corneal opacities & 8 \\
Adherent leucoma & 4 \\
Pterygia & 4 \\
Cataracts & 3 \\
Disc pallor & 2 \\
Phthisis bulbi & 1 \\
Aphakia & 1 \\
\hline
\end{tabular}

TABLE VIII Detection of antibody titres to Ad-4 and enterovirus type-70 (23356/71 and EH91/71) in 35 serum samples collected during the first visit

\begin{tabular}{llll}
\hline Reciprocal & \multicolumn{3}{l}{ No. of patients } \\
\cline { 2 - 4 } Antibody titre & Ad-4 & $23356 / 71$ & EH91/7I \\
\hline$<20$ & 15 & 11 & 12 \\
20 & 7 & 10 & 10 \\
40 & 7 & 8 & 9 \\
80 & 4 & 4 & 3 \\
160 & 2 & 2 & 2 \\
\hline Total & 35 & 35 & 35 \\
\hline
\end{tabular}

TABLE IX Detection in rise of antibody titres to adenovirus type 4 (Ad-4) and enterovirus type 70 (i) EV-70/23356/71; (ii) $E V-70 / E H 91 / 71)$ in 15 paired serum samples

\begin{tabular}{llll}
\hline & \multicolumn{4}{l}{ No. of serum pairs } \\
\cline { 2 - 4 } Rise in Ab titre & Ad-4 & $23356 / 71$ & EH91/71 \\
\hline None & 14 & 9 & 9 \\
2-fold & 1 & 4 & 4 \\
4-fold & 0 & 2 & 2 \\
\hline Total & 15 & 15 & 15 \\
\hline
\end{tabular}

significant difference between the antibody response to the London or the Singapore isolate of EV-70 in 15 paired serum samples (Table 9). Two pairs of sera showed a 4-fold rise from $<1: 20$ to $1: 40$, whereas four pairs of sera showed a 2-fold rise in titre to EV-70. The rest of the pairs showed no rise in antibody titres. Only one pair of serum samples had a 2 -fold rise in antibody levels to adenovirus.

A 4-fold or greater rise in antibody titre or a static titre greater than $1: 40$ to $\mathrm{EV}-70$ was considered to indicate a recent infection. Hence the two pairs of sera which had a 4-fold rise in titre to EV-70 and the six sera which had a titre greater than 1:40 to London isolate were regarded as positive. The four sera which had a titre greater than 1:40 to Singapore isolate of EV-70 had the same levels of antibodies to the London isolate of EV-70. All the sera were put through the test system a second time to confirm the results obtained initially. 


\section{Discussion}

The sequence of events during the epidemic of AHC in Northern Nigeria in 1985 indicates that the epidemic may have started in the girl's boarding school in Kaduna and from there spread rapidly to the urban population. By early 1986, the epidemic had extended to Southern Nigeria. At the school, AHC spread rapidly owing to over-crowding of the dormitories, inadequate sanitation, shortage of water, and the unhygienic habits of the students. Following the first examination and at the height of the epidemic the students were sent home because it was the end of the school term. The release of infected students into the community was probably responsible for the community outbreak of AHC.

Students, industrial workers, housewives, and office workers formed the main groups of people who acquired the infection. Only one patient was from the rural area. The Guinness Ophthalmic Clinic is the only well established 'public' eye clinic in Northern Nigeria, and it serves a large number of villagers with ophthalmic problems, including conjunctivitis. Hence either the AHC epidemic was mainly restricted to the urban population with no sizeable epidemic in the relevant rural population, or villagers with $\mathrm{AHC}$ did not attend the clinic.

The clinical feature and severity of AHC observed in our patients were very similar to those reported elsewhere ${ }^{1-5}$ except for the presence of SPK in $56.4 \%$ of the cases compared with $3.9 \%$ reported in an earlier episode of AHC. ${ }^{8}$ Coalescent corneal erosions were observed in $5.9 \%$ of the total cases. The erosion was limited and did not affect vision. During the short period of follow-up in our patients we did not see any evidence of neurological manifestation. Neurological disorders have been reported during epidemics of AHC, particularly in Asia. ${ }^{9}$

The adverse effect of self-medication on the prognosis of AHC has been reported previously. ${ }^{10}$ About $46 \%$ of the patients we saw had been treated previously. One patient had applied a mixture of locally brewed gin ogogoro and sugar water. This resulted in severe corneal melting and corneal vascularisation.

Serological studies, especially tests for neutralising antibodies, have been used extensively to investigate the role of $\mathrm{EV}-70$ as the causative agent of AHC. The tests are particularly useful where facilities for isolation of EV-70 do not exist. This was experienced during an outbreak of AHC in 1981 in India ${ }^{11}$ and Zaire. ${ }^{12}$

Fifteen paired and 20 single serum samples were available for serological studies. In our laboratory we have found a good correlation in antibody levels to EV-70 in sera by using either a neutralisation method or an indirect immunofluorescence test (unpublished data). The antibody response to the London isolate and the Singapore isolate of EV-70 was not significantly different. Only two pairs of sera showed a 4-fold rise in antibody levels to EV-70, whereas the titres to EV-70 in the rest of the sera ranged from $1: 20$ to $1: 160$. This made it rather difficult for us to interpret the results. Neutralising antibody levels of $1: 16$ or greater to EV-70 in convalescent sera has been considered to be a critical diagnositic level. ${ }^{13}$ However, neutralising antibody titres from 1:16 and 1:64 against EV-70 have also been shown to persist in sera for up to 21 months. ${ }^{21}$ Hence, there is always a possibility that the presence of these antibodies may have been due to a previous exposure to the agent. In $136(68 \%)$ of our patients a previous history of severe conjunctivitis was reported. We therefore considered a titre of greater than 1:40 as a good indicator of recent infection.

The sera were also screened for the presence of antibody levels to adenovirus. None of the paired serum samples showed a 4-fold rise in antibody. Six sera (three paired serum samples) had a static titre greater than 1:40 to adenovirus. This could be due to a previous exposure to ocular or pharyngeal infection with adenovirus. There is also a possibility that these three patients were cases of adenovirus ocular infection which were clinically indistinguishable from that of EV-70.

Many epidemiological and laboratory problems associated with the study of epidemics of acute haemorrhagic conjunctivitis still remain unsolved. Perhaps the development of more sensitive culture tests for the isolation of causative agents or direct methods for the detection of virus in clinical specimens, along with complementary serological tests, will help us to understand epidemics of acute haemorhagic conjunctivitis.

1 Chatteriee S, Quarcoopome CO, Apenteng A. An epidemic of acute conjunctivitis in Ghana. Ghana Med f 1970; 9: 1-11.

2 Chatterjee S, Quarcoopome CO, Apenteng A. Unusual type of epidemic conjunctivitis in Ghana. BrF Ophthalmol 1970; 54: 628-30.

3 Anonymous. Acute haemorrhagic conjunctivitis. $\mathrm{Br} \mathrm{Med} \mathcal{J}$ 1982; 284: 833-4.

4 Akinsete EO. Epidemic conjunctivitis in Lagos, Nigeria. $f$ Nigeria Med Assoc 1970; 1: 46-50.

5 Salami MYI. Pattern of epidemic conjunctivitis among the school age population in Lagos. $\mathcal{F}$ Nigeria Med Assoc 1971; 1 . 184-8.

6 Mikrovic RR, Schmidt NJ, Yin-Murphy M, Melnick JL. Enterovirus etiology of the 1970 Singapore epidemic of acute conjunctivitis. Intervirology 1974; 4: 119-27.

7 Yin-Murphy M, Lim KH, Chupa PH. Adenovirus type 11 epidemic conjunctivitis in Singapore, Southeast Asia. Asia f Trop Med Publ Health 1974; 5: 333-41.

8 WHO Weekly Epidemiological Record 1981; 56: 254.

9 John TJ, Christopher S, Abrahams J. Neurological manifestations of acute haemorrhagic conjunctivitis due to enterovirus-70. Lancet 1981; ii: $1283-4$.

10 McMoli TE, Bordoh AN, Munube GMR, Bell EJ. Epidemic acute haemorrhagic conjunctivitis in Lagos, Nigeria. $\mathrm{Br} \mathcal{F}$ Ophthalmol 1984;68: 401-4.

11 Kono R, Migamura K, Ogino T, et al. 1981. Antibody titres to enterovirus-70 in the 1981 Indian epidemic of acute enterovirus-70 in the 1981 Indian epidemic of

12 Desmyter J, Colaert J, Maertens K, Muyembe T. Enterovirus 70 haemorrhagic conjunctivitis in Zaire, 1981 versus 1972 Lancet 1981; ii: 1054-5.

13 Kono R, Sasagawa A, Miyamura K, Tajiri E. Serologic characterisation and sero-epidemiologic studies on acute haemorrhagic conjunctivitis (AHC) virus. Am $\mathcal{F}$ Epidemio 1975; 101: 444-57.

14 Kono R, Miyamura K, Tajiri E, et al. Neurologic complications associated with acute hemorrhagic conjunctivitis virus infection and its serologic confirmation. F Infect Dis 1974; 129: $590-3$. 